\title{
Baseline Power of Theta Oscillations Predicts Mystical-Type Experiences Induced by DMT in a Natural Setting
}

\author{
Enzo Tagliazucchi ${ }^{1,2 * t}$, Federico Zamberlan ${ }^{1 \dagger}$, Federico Cavanna ${ }^{1,3}$, Laura de la Fuente ${ }^{1}$, \\ Celeste Romero ${ }^{4}$, Yonatan Sanz Perl ${ }^{1,5}$ and Carla Pallavicini ${ }^{1,3}$ \\ ${ }^{1}$ Departamento de Física, Universidad de Buenos Aires and Instituto de Física de Buenos Aires (IFIBA-National Scientific and \\ Technical Research Council (CONICET)), Buenos Aires, Argentina, ${ }^{2}$ Latin American Brain Health Institute, Universidad Adolfo \\ Ibanez, Santiago, Chile, ${ }^{3}$ Fundación Para la Lucha Contra las Enfermedades Neurológicas de la Infancia, Buenos Aires, \\ Argentina, ${ }^{4}$ Centro de Estudios de la Cultura Cannábica, Buenos Aires, Argentina, ${ }^{5}$ Center for Brain and Cognition, \\ Computational Neuroscience Group, Universitat Pompeu Fabra, Barcelona, Spain
}

OPEN ACCESS

Edited by:

Graham Campbell,

Hammersmith Medicines Research United Kingdom

Reviewed by:

Daryl Wayne Niedermoser,

University Psychiatric Clinic

Basel, Switzerland

Ivan V. Brak,

State Scientific Research Institute of

Physiology and Basic Medicine, Russia

*Correspondence: Enzo Tagliazucchi enzo@df.uba.ar

${ }^{\dagger}$ These authors have contributed equally to this work

Specialty section

This article was submitted to

Psychological Therapies,

a section of the journal

Frontiers in Psychiatry

Received: 08 June 2021 Accepted: 07 October 2021 Published: 05 November 2021

Citation:

Tagliazucchi E, Zamberlan F Cavanna F, de la Fuente L, Romero C, Sanz Perl Y and Pallavicini C (2021) Baseline Power of Theta Oscillations Predicts Mystical-Type Experiences Induced by DMT in a Natural Setting.

Front. Psychiatry 12:720066.

doi: 10.3389/fpsyt.2021.720066
$\mathrm{N}, \mathrm{N}$-Dimethyltryptamine (DMT) is a classic psychedelic capable of inducing short-lasting but profound changes in consciousness. As with other psychedelics, the experience induced by DMT strongly depends upon contextual factors, yet the neurobiological determinants of this variability remain unknown. The present study investigated changes in neural oscillations elicited by inhaled DMT, and whether baseline electroencephalography (EEG) recordings could predict the subjective effects reported by the participants. Healthy volunteers $(N=35)$ were measured with EEG before and during the acute effects of DMT consumed in a natural setting. Source-localized neural oscillations were correlated with the results of multiple questionnaires employed to assess the subjective effects of the drug. DMT resulted in a marked reduction of alpha and beta oscillations, and increased posterior spectral power in the delta, theta and gamma bands. The power of fronto-temporal theta oscillations was inversely correlated with scales indexing feelings of unity and transcendence, which are an integral part of the phenomenology of mystical-type experiences. The robustness of these results was supported using a machine learning model for regression trained and tested following a cross-validation procedure. These results are consistent with the observation that the state of mind prior to consuming a psychedelic drug influences the ensuing subjective experience of the user. They also suggest that baseline EEG screenings before administration of a serotonergic psychedelic could be useful to estimate the likelihood of inducing mystical-type experiences, previously linked to sustained positive effects in well-being and improved outcome of therapeutic interventions.

Keywords: psychedelics, DMT, EEG, neural oscillations, mystical-type experiences

\section{INTRODUCTION}

Serotonergic psychedelics (a subset of 5- $\mathrm{HT}_{2 \mathrm{~A}}$ receptor agonists) are capable of inducing remarkable changes in perception, affect, self-awareness and cognition (1). Some of the effects elicited by psychedelics are long-lasting, for instance, changes in personality traits and improvements in the symptoms of certain mental health conditions (2-4). After decades of 
neglect, psychedelics have resurfaced into the mainstream of human neuroscience and psychiatry, a revolution spearheaded by neuroimaging studies in healthy volunteers (5) and clinical research that supports the efficacy of these drugs for the treatment of depression (6-8), substance abuse (9), and existential anxiety $(10,11)$, among other uses. These advances, combined with the excellent safety profile of all classic psychedelics [mescaline, psilocybin, lysergic acid diethylamide (LSD) and $\mathrm{N}, \mathrm{N}$-dimethyltryptamine (DMT)] (12), resulted in a surge of scientific studies aimed to understand how psychedelics exert their effect on consciousness and how these effects relate to their therapeutic mechanism of action (13).

The capacity of psychedelics to induce long-lasting psychological effects is related to the qualities of the subjective experience during the acute effects of the drug. When administered in a pleasant and supportive setting, psilocybin is capable of inducing profoundly meaningful experiences of deep personal significance, with a sustained positive effect in wellbeing and behavior $(14,15)$. The subjective experience linked to these transformative properties of psychedelics has substantial commonalities with other non-ordinary states of consciousness, known in the previous literature as mystical experiences, conversion experiences, peak experiences, transcendental experiences, epiphanies, among other denominations $(16,17)$. Because of these similarities, Griffiths and colleagues introduced the name "mystical-type" to refer to these experiences, and built upon previous work to create the Mystical Experience Questionnaire (MEQ30), with a total of 30 items leading to four subscale scores (mystical, positive mood, transcendence of time and space, and ineffability) (18). Psilocybin-induced mystical-type experiences are dose dependent (19) and are related to the therapeutic effects of the drug when administered in patients suffering from depression (20), end of life anxiety (11) and substance abuse disorders (21), thus prompting the need to understand how these experiences can be predicted and facilitated.

Considerable variability exists in the percentage of subjects whose report qualifies as a complete mystical-type experience, according to the criteria put forward by Griffiths and colleagues. These percentages range from $60 \%$ for multiple studies using psilocybin $(16,17)$ to $10 \%$ for LSD $(22)$, and between 37 and $73 \%$ for intravenous or inhaled DMT $(23,71)$. This variability is likely related to contextual factors known as set and setting, i.e., the state of mind of the participant prior to the experience and the environment where the experience takes place, respectively (24). For instance, Haijen et al. conducted a large online survey to predict the response to psychedelic drugs, finding that trait absorption and clear intentions are conducive to mystical-type experiences (25). A recent metaanalysis identified other predictors of mystical-type experiences, such as age, apprehension, deservingness, spiritual motivations, surrender, acceptance and attachment anxiety (26). Most predictive variables explored to date were obtained from selfreported measures assessed using standardized questionnaires and meta-analyses (25-27). However, insofar as the state of mind of the user is reflected in baseline measurements of brain activity, these measurements should also present predictive power about the ensuing psychedelic experience, potentially informing the neurobiological mechanisms underlying the repertoire of possible responses to psychedelic drugs. Notably, the development of predictive models based on brain activity recordings remains heavily underexplored in comparison to those based on psychometric data.

We explored baseline EEG oscillations as predictors of selfreported subjective effects in a cohort of 35 subjects who inhaled DMT in freebase form in their preferred context of use. DMT is frequently consumed in ceremonial settings, where it is crystallized over non-psychoactive plant leaves and then inhaled after combustion $(28,29)$, leading to intense but short-lasting subjective effects (30-32). We chose to investigate DMT under natural conditions to encompass an ample range of sets and settings, an advantage when searching for contextual factors that are linked to specific drug-induced experiences $(24,33)$. Our main result is a relationship between mystical-type experiences and baseline theta oscillations originating from frontal and temporal brain regions.

\section{MATERIALS AND METHODS}

The data included in this manuscript was used in a previous publication, which can be referenced for further methodological details (71).

\section{Participants}

Thirty-five participants ( 7 females, $33.1 \pm 6$ years, $92.2 \pm$ 201.4 previous experiences with ayahuasca, $3.6 \pm 5.6$ previous experiences with DMT alone) were recruited by word-of-mouth and social media advertising between May and December 2019.

Participants were required to have at least two previous experiences with ayahuasca or DMT, abstain from consuming psychoactive drugs at least $24 \mathrm{~h}$ prior to the study, and be willing to engage in their preferred use of DMT in the presence of research team members. Researchers did not provide DMT or other psychoactive compounds to the subjects, interacted with their use of the substance, nor promoted the use of DMT or other psychedelics in any way. They role was only observational, a requirement for participation in the experiment was that subjects conducted their use of DMT in their preferred context and as previously planned. To participate, subjects had to be between 21 and 65 years old.

Subjects were excluded due to reported past difficult experiences with psychedelics, and based on the results of a non-diagnostic interview (SCID-CT, First, 2014) conducted with psychiatrist, according to the guidelines by Johnson et al. (12). Subjects who fulfilled DSM-IV criteria for the following disorders were excluded from the experiment: schizophrenia or other psychotic disorders, and type 1 or 2 bipolar disorder (both also in first and second degree relatives), substance abuse or dependence over the last 5 years (excluding nicotine), depressive disorders, recurrent depressive episodes, obsessive-compulsive disorder, generalized anxiety disorder, dysthymia, panic disorder, bulimia or anorexia, as well as subjects with history of neurological disorders. Subjects who presented one standard deviation above the mean in the State-Trait Anxiety Inventory (34) were 
excluded, as well as subjects under psychiatric medication of any kind.

This study was conducted in accordance with the Helsinki declaration and approved by the Committee for Research Ethics at the Jose Maria Ramos Mejia General Hospital (Buenos Aires, Argentina), accredited by the Central Committee of Ethics, Ministry of Health of the Autonomous City of Buenos Aires, Argentina.

All research data associated with this manuscript is publicly available without restrictions (10.5281/zenodo.3992359).

\section{DMT Administration}

Subjects consumed DMT in their preferred context of use. After being fitted with the EEG cap, the subjects were instructed to keep their eyes closed, relax and maintain an upright position, avoiding head movement and jaw clenching to prevent muscle artifacts. Only four subjects self-administered DMT, all others were assisted by facilitators, who organized the retreats or ceremonies attended by the participants. After receiving instructions by the facilitators, subjects inhaled the smoke and vapor resulting from the combustion of freebase DMT, in all cases recrystallized over non-psychoactive plant leaves. Facilitators withdrew the pipe when subjects either stopped inhaling and leaned back, or exhausted the contents of the pipe. In all cases, the load of the pipes was estimated by the participants or their facilitators at around $40 \mathrm{mg}$ freebase DMT, and reported to be extracted from the root of Mimosa hostilis (also known as Mimosa tenuiflora or jurema) (35). The presence of DMT was verified in all samples by high performance liquid chromatography coupled to mass spectrometry for profiling and qualitative analysis.

\section{Psychometric Questionnaires}

Before the DMT experience, participants completed Spanish versions of the State Trait Anxiety Inventory (STAI trait) (34), and questions introduced in Haijen et al. (25) to assess the selfreported adequateness of set, setting and intentions. After the DMT experience, participants completed the $5 \mathrm{D}$ altered states of consciousness scale (5D-ASC) (36), the mystical experience questionnaire (MEQ30) (18), the near-death experience scale (NDE) (37), and a series of questions to assess the impact of set, setting and social interactions on the psychedelic experience (post-experience questionnaire, or "Post"). Immediately before and after the DMT experience, participants completed Spanish versions of the Big Five personality (BFI) test (38), STAI state (34), and Tellegen absorption scale (TAS) (39).

\section{EEG Acquisition and Pre-processing}

EEG data were recorded with a 24-channel mobile system (mBrainTrain LLC, Belgrade, Serbia, http://www.mbraintrain. $\mathrm{com} /$ ) attached to an elastic electrode cap (EASYCAP GmbH, Inning, Germany, www.easycap.de). Twenty-four $\mathrm{Ag} / \mathrm{AgCl}$ electrodes were positioned at standard 10- 20 locations (Fp1, Fp2, Fz, F7, F8, FC1, FC2, Cz, C3, C4, T7, T8, CPz, CP1, CP2, CP5, CP6, TP9, TP10, Pz, P3, P4, O1, and O2). Reference and ground electrodes were placed at $\mathrm{FCz}$ and $\mathrm{AFz}$ sites. The wireless EEG DC amplifier (weight $=60 \mathrm{~g}$, size $=82 \times 51 \times 12 \mathrm{~mm}$, resolution $=24$ bit, sampling rate $=500 \mathrm{~Hz}, 0-250 \mathrm{~Hz}$ pass-band) was attached to the back of the electrode cap (between electrodes $\mathrm{O} 1$ and $\mathrm{O} 2$ ) and sent digitized EEG data via Bluetooth to a Notebook held by a experimenter sitting behind the participant. Prior to the administration of DMT, baseline EEG activity was acquired with eyes open and closed (5 min each). After the DMT was administered, EEG recordings started when subjects exhaled, and lasted until the subject indicated a return to baseline $(6 \pm$ $1.4 \mathrm{~min})$.

EEG data was preprocessed using EEGLAB (https://sccn. ucsd.edu/eeglab/index.php) (40). Data was divided into $2 \mathrm{~s}$ epochs, then bandpass-filtered $(1-90 \mathrm{~Hz})$ and notch-filtered $(47.5-52.5 \mathrm{~Hz})$. Artifact-laden channels were first detected using EEGLAB automated methods. All channels were manually inspected before rejection (mean 30\% rejected channels, max. 8 channels) and then interpolated using data from the surrounding channels. Epochs to be rejected were flagged automatically and in all cases removed after manual inspection $(21.3 \pm 13.7$ epochs rejected per subject). Infomax independent component analysis (ICA) was then applied to data from each individual participant, and used to manually identify and remove artifactual components $(2.7 \pm 1.1$ components removed). According to the previously defined criteria, 6 subjects were discarded from the subsequent EEG analysis due to an excessive number of rejected epochs and/or channels, resulting in 29 subjects for subsequent analysis.

\section{Source EEG Power Estimation}

Source imaging analysis was performed using Brainstorm (https://neuroimage.usc.edu/brainstorm/) (41). Pre-processed EEG epochs were imported and localized to a standard MRI volume divided by a $4 \mathrm{~mm} 3 \mathrm{D}$ grid. A 3 -shell sphere model was used to compute the forward solutions, with adaptive integration and standard tissue conductivities. Sources were estimated by minimum norm imaging with unconstrained dipole orientations and identity noise covariance matrix, yielding a current density map normalized by the square root of the estimated noise variance at each location in the map, i.e., a z-score statistical map (dynamical Statistical Parametric Mapping, dSPM) (42).

Band-specific power spectral density was computed using the Welch method followed by spectrum normalization and averaging across epochs. Spectral power was computed for the following frequency bands: delta $(1-4 \mathrm{~Hz})$, theta $(4-8 \mathrm{~Hz})$, alpha $(8-12 \mathrm{~Hz})$, beta $(12-30 \mathrm{~Hz})$, low gamma $(30-40 \mathrm{~Hz})$, and high gamma $(40-70 \mathrm{~Hz})$. Finally, source spectral power estimates were averaged across all grid locations within the cortical and subcortical regions defined in the Automated Anatomical Labeling (AAL) atlas, yielding 90 values per subject and frequency band (43).

\section{Statistical Analyses}

Significant differences in regional mean spectral power between conditions were assessed using Wilcoxon's non-parametric signed-rank test. The predictive power of baseline activity was evaluated using Spearman's non-parametric rank correlation coefficient between regional spectral estimates during the eyes closed condition and the results of questionnaires (5D-ASC, $\mathrm{NDE}$, and MEQ30) obtained after the DMT condition. 

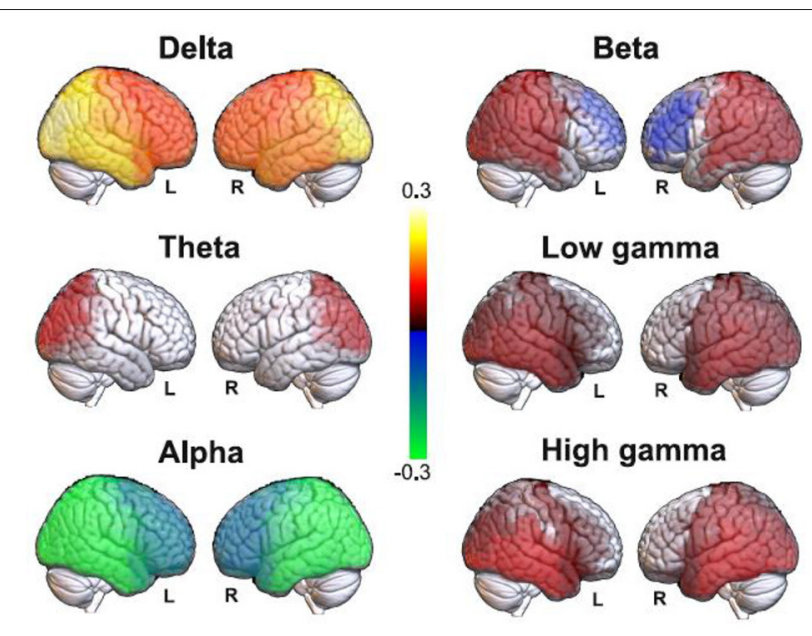

FIGURE 1 | Significant differences in band-specific source EEG power between the eyes closed baseline and the DMT condition. Red-yellow and blue-green color scales indicate increased and decreased spectral power under DMT compared to the baseline, respectively.

The Benjamini-Hochberg procedure was followed to control for the false discovery rate, setting a rate of $\alpha=0.05$ (44). Correlation $p$-values obtained for all frequency bands and questionnaire subscales were pooled together before the application of this procedure.

\section{Machine Learning Regression Model}

A random forest regressor (45) with 1,000 estimators (implemented in scikit-learn, https://scikit-learn.org/) (46) was trained using $50 \%$ of the samples to estimate the $5 \mathrm{D}$-ASC Unity, MEQ30 Mystical and MEQ30 Transcendence scores in the remaining 50\% (randomly selected for each independent instance of the classifier). Each sample consisted of the theta power source values at the $90 \mathrm{AAL}$ regions for a participant during the eyes closed baseline. This procedure was repeated 1,000 times for each questionnaire subscale with and without shuffling of the target scores. Finally, a $p$-value was constructed by counting the number of times the mean squared error of the randomized classifier was lower than that of the classifier trained using the non-shuffled scores, divided by the total number of iterations.

\section{RESULTS}

\section{Differences in Source EEG Power Between DMT and Baseline}

We first compared the EEG source estimates for all frequency bands between the eyes closed and DMT conditions, with the purpose of estimating the validity of the source imaging procedure by comparing the results with analyses previously conducted at the scalp level (71). The results of this analysis are shown in Figure 1. We observed posterior increases in delta power under the acute effects of DMT, with similar (but less marked) changes for the theta band. For the alpha band we observed changes similar to those seen in the delta band, but of opposite sign (i.e., alpha power decreases in occipito-temporal regions under DMT). Beta power increased in posterior regions and decreased in prefrontal regions, while occipital, parietal and temporal gamma increases appeared under DMT. These results are consistent with those previously reported at the scalp level (71), but with some additional significant differences in theta and beta bands.

\section{Correlations Between Baseline Activity and Questionnaire Subscales}

We computed all correlations between regional spectral power estimates and questionnaire subscales. We first conducted this analysis at the scalp level, finding significant negative correlations between theta power and the subscales 5D-ASC Experience of unity ("Unity"), MEQ30 Mystical experience ("Mystical”) (in both cases electrodes Fp1, Fp2, F7, F8, Fz, T7, T8), and MEQ30 Transcendence of time and space ("Transcendence") (electrodes Fp1, Fp2, F7, F8, Fz, FC1, FC2).

Next, we applied the same analysis to the source theta power, with the objective of visualizing these changes overlaid on brain anatomy. After FDR-correction for multiple comparisons, we only identified significant correlations for the theta band (see Figure 2 for a matrix summarizing all correlations for this frequency band). These significant correlations corresponded to the following three subscales: 5D-ASC Unity ("Unity"), MEQ30 Mystical, and MEQ30 Transcendence (i.e., the same subscales that presented significant results at the scalp level).

Figure 3 presents the anatomical rendering and volumetric display of the AAL regions whose baseline theta power presented significant correlations with 5D-ASC Unity, MEQ30 Mystical, and MEQ30 Transcendence. Consistent with the analysis at the scalp electrodes, 5D-ASC Unity was inversely correlated with theta power at fronto-temporal regions, and a similar pattern of significant correlations was observed for MEQ30 Mystical, while negative correlations between theta power and MEQ30 Transcendence were distributed frontally and bilaterally, spanning the prefrontal cortex, the orbital part of the frontal lobe and the anterior cingulate cortex.

\section{Prediction Using a Cross-Validated Machine Learning Regression Model}

Figure 4 shows the prediction of individual questionnaire subscale values based on the theta power localized at the 90 AAL regions, with the $y$-axis representing the predicted values (obtained using a random forest regression model) and the $\mathrm{x}$-axis representing the actual values. In both cases, values were standardized to $\mathrm{z}$-scores before visualization. As shown in the insets, the predicted values presented a medium to large correlation with the actual values, however, the model tended to underestimate the scores that were significantly smaller than the mean (i.e., largest negative values after conversion to z-scores).

\section{Prediction Based on Psychometric Data}

We computed the correlation between 5D-ASC Unity, MEQ30 Mystical and MEQ30 Transcendence subitem scores, and baseline psychometric scores including personality traits (BFI), 


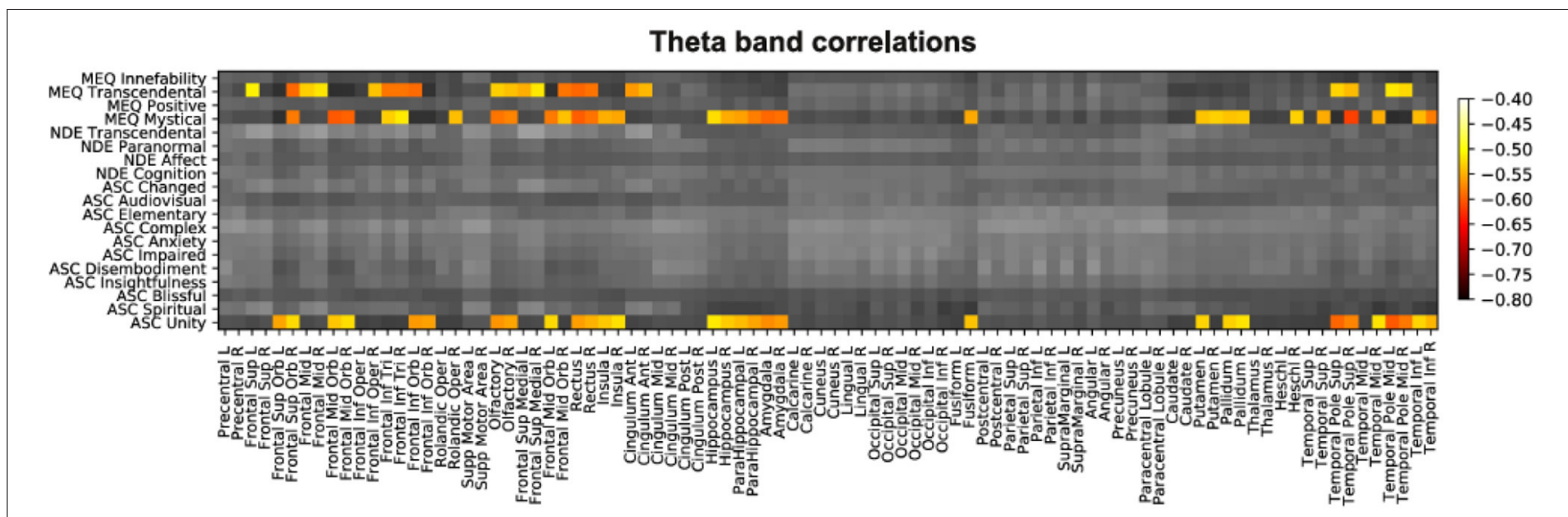

FIGURE 2 | Matrix with all correlations between questionnaire subscales (y-axis) and source theta power computed for all AAL regions ( $\mathrm{x}$-axis). Significant correlations were in all cases negative and are indicated by colored entries.
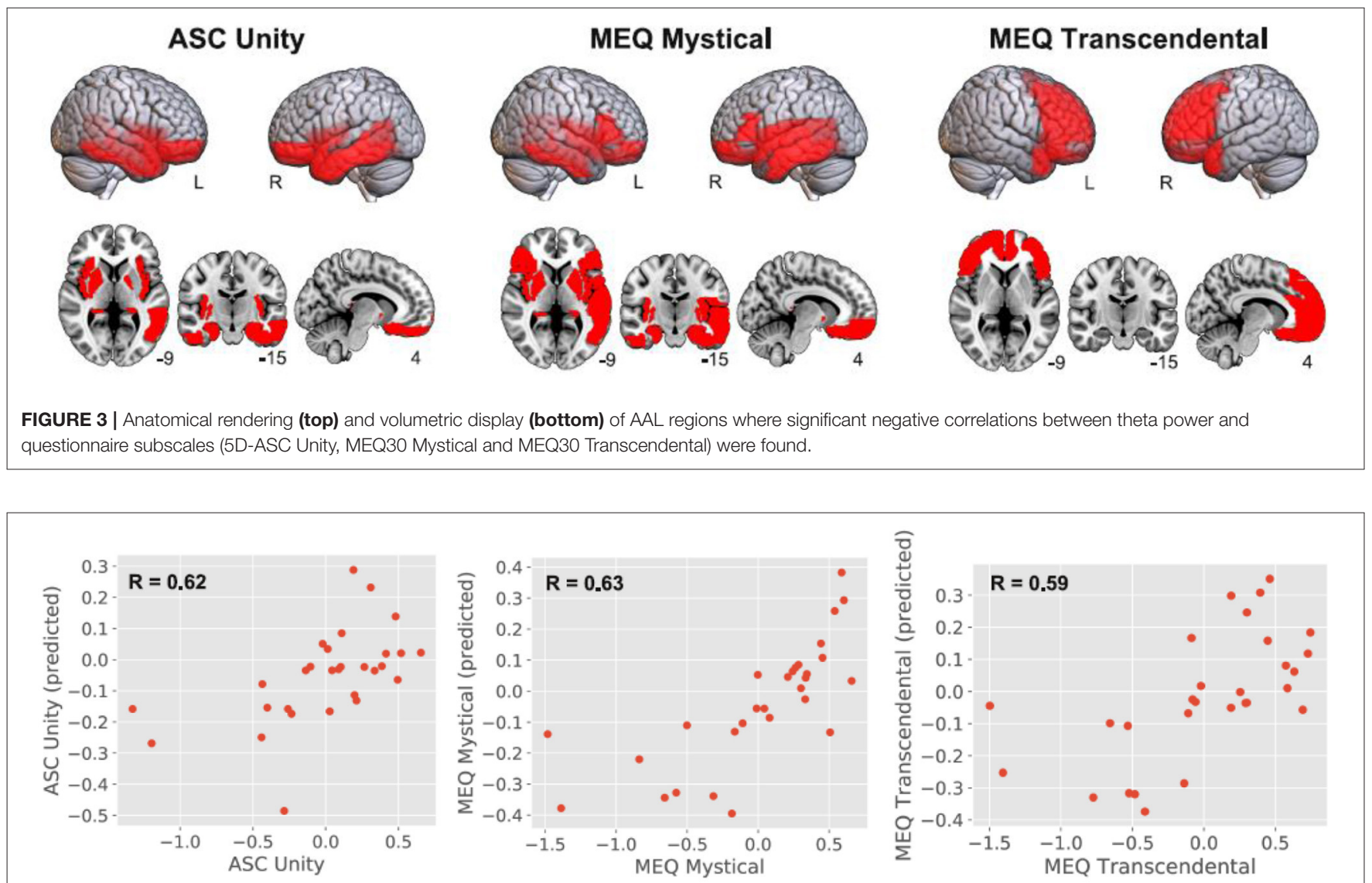

FIGURE 4 | Predicted (random forest regression model) vs. actual questionnaire subscale values (in both cases standardized to z-scores) with the linear correlation coefficient between both included as an inset.

trait anxiety (STAI), and trait absorption (TAS). Consistent with a previous survey study (25), we only found significant correlations with trait absorption $(R=0.43$ for $5 \mathrm{D}$-ASC Unity and $R=0.51$ for MEQ30 Mystical).

\section{DISCUSSION}

We investigated how the acute effects of DMT modified source-localized EEG spectral power, as well as the relationship 
between baseline spectral power fluctuations and the conscious experience reported by the subjects. Our recordings were obtained in natural settings, i.e., the contexts preferred by the participants for their use of DMT. While this approach is limited in many ways compared to a double-blind placebocontrolled design, it also presents distinct advantages, mainly related to an ample range of settings. These advantages are especially important when attempting to predict the response to DMT from baseline brain activity measurements $(24,33)$. We followed a novel approach, diverging from previous efforts to predict the effects of psychedelics from questionnaires or from natural language descriptions of set and setting (25-27). Since the mindset of the participants should be reflected on their baseline neural oscillations, we hypothesized that band-specific EEG power could predict some of the subjective effects reported by them after the DMT experience.

Our results at the source level are consistent with a previous analysis of this data (71) and with an independent report of EEG changes elicited by intravenous DMT administration (47). The most salient results obtained from the contrast of DMT vs. eyes closed comprise increased delta power and decreased alpha power, in both cases localized to posterior brain regions, replicating previous analyses. Also, we found that DMT increased the power of gamma oscillations in occipital and temporal regions, a similar increase of oscillations in the gamma range was previously reported by our group, as well as by others investigating orally administered DMT in the form of ayahuasca (48). However, we also found new significant changes in the theta and beta frequency bands, suggesting that mixed sources registered at the scalp could hinder the level of significance in the comparison of DMT vs. eyes closed.

Theta oscillations have been linked to multiple cognitive and perceptual processes in the healthy human brain, some of them subserving mind-wandering (49-52). We speculate that these functions could be detrimental for a state of mind conducive to mystical-type experiences, an observation supported by the facilitation of these experiences by mindfulness training (53). Increased theta power has been reported during mind-wandering episodes, especially when divided by beta power to form the beta/theta ratio $(54,55)$. Theta oscillations have been linked to episodes of self-assessed (i.e., subjective) drifts of attention during a breath counting task (56), as well as to behaviorally indexed mind-wandering (57). Dissociative absorption, a trait predictive of psychedelic-induced mystical-type experiences, correlates inversely with the power of theta rhythms originating within the temporal lobes (58). A combined EEG-fMRI study established that intrusions of self-generated and internally-oriented thought processes are characterized by reduced default mode network activity and increased power of theta oscillations, among other frequency bands (59). Anxiety prior to the DMT experience could hinder the likelihood of undergoing a mystical-type experience, which is consistent with the reported link between anxious rumination and increased theta power localized to the anterior cingulate cortex (60).

Oscillations in the theta band are thought to reflect top-down influences related to working memory and memory retrieval (49-52), and are linked to the activation of autobiographical memories (61) as well as to mental time travel (62) and, more generally, to the level of mental workload (63). While these aspects of theta oscillations could be related to mindwandering, multiple reports show enhanced theta power in mindfulness meditators, as well as in meditators following other contemplative traditions (64-67). Furthermore, frontal theta oscillations are inversely correlated with default mode activity, suggesting that these oscillations signal episodes of sustained attention (68). These apparent contradictions could stem from the lack of a unique source for the theta rhythm, with different sources reflecting equally distinct cognitive roles, and from the possibility of rhythms of the same frequency but different functional roles emerging from the same cortical regions (69). Since we did not assess the prevalence of mind-wandering episodes prior to the DMT experience, we lack subjective or behavioral validation for the proposed disruptive role of theta oscillations concerning mystical-type experiences. However, we note that high absorption is predictive of these experiences (as previously shown and also confirmed by our analysis) and at the same time indicative of deep and effortless concentration (70) and inversely correlated with EEG theta power (58), thus lending indirect support to the hypothesized origin of our main results.

Our analysis failed to predict other aspects of the psychedelic experience, such as perceptual, affective or cognitive alterations. Haijen et al. showed that the intensity of visual effects could be predicted by absorption, dose, and by having clear intentions for the experience (25). However, other studies suggest that predicting perceptual modifications from non-pharmacological variables is more difficult than predicting mystical-type experiences (27). A recent meta-analysis established that baseline psychometric questionnaires are mostly predictive of affective or mystical-type experiences, with only sparse significant associations of baseline variables with perceptual or cognitive modifications (26). We can speculate that individual characteristics that are beyond the spatial resolution of EEG could underlie the variability in other dimensions of the psychedelic experience, for instance, differences in the cortical density of serotonin 5-HT2A receptors. Future studies should attempt to predict the response to psychedelics from more exhaustive neuroimaging recordings, including functional, anatomical, and neurochemical data.

The spatial accuracy of source imaging is very limited for the number of electrodes used in our recordings. However, we did not draw conclusions based on individual AAL regions, but only at the coarser level of brain lobes and hemispheres, which were in all cases consistent with results obtained at the scalp level. In this sense, source imaging is useful to present findings overlaid on brain anatomy, which facilitates direct visual identification of the involved cortical regions, but should not be overinterpreted in terms of spatial localization. Another limitation of our study is the lack of a placebo condition to control for expectancy effects, as well as the limited information concerning the administered doses of DMT. These limitations are inherent to studies conducted in natural settings and thus future research should attempt to reproduce our findings following a randomized double-blind placebo-controlled design. However, we note that predicting how contextual factors influence the acute 
effects of psychedelics might be difficult if variables related to set and setting are uniform across participants. In this sense, a certain degree of contextual heterogeneity (as in the case of naturalistic studies) could represent an advantage instead of a limitation.

In summary, our study represents a first step in the direction of predicting the acute effects of psychedelics from baseline neurophysiological recordings. Although debated, the therapeutic properties of psychedelics seem to depend upon the state of consciousness manifested during the experience itself. As more clinical research is conducted and more data becomes available, we expect that certain profiles of subjective effects will be associated with the improvement of patients after treatment with psychedelics. Ultimately, the field will face the problem of how to engineer these desirable subjective effects, a problem that will require a systematic exploration of the response to psychedelic drugs from baseline psychological and neurophysiological measurements.

\section{DATA AVAILABILITY STATEMENT}

The datasets presented in this study can be found in online repositories. The names of the repository/repositories and accession number(s) can be found below: doi: 10.5281/zenodo.3992359.

\section{REFERENCES}

1. Nichols DE. Psychedelics. Pharmacol Rev. (2016) 68:264355. doi: 10.1124/pr.115.011478

2. Bouso JC, Dos Santos RG, Alcázar-Córcoles MÁ, Hallak JE. Serotonergic psychedelics and personality: a systematic review of contemporary research. Neurosci Biobehav Rev. (2018) 87:118-32. doi: 10.1016/j.neubiorev.2018.02.004

3. Aday JS, Mitzkovitz CM, Bloesch EK, Davoli CC, Davis AK. Long-term effects of psychedelic drugs: a systematic review. Neurosci Biobehav Rev. (2020) 113:179-89. doi: 10.1016/j.neubiorev.2020.03.017

4. Nutt D, Carhart-Harris R. The current status of psychedelics in psychiatry. JAMA Psychiatry. (2021) 78:121-2. doi: 10.1001/jamapsychiatry.2020.2171

5. Dos Santos RG, Osorio FL, Crippa JAS, Hallak JE. Classical hallucinogens and neuroimaging: a systematic review of human studies: Hallucinogens and neuroimaging. Neurosci Biobehav Rev. (2016) 71:715-28. doi: 10.1016/j.neubiorev.2016.10.026

6. Carhart-Harris RL, Bolstridge M, Rucker J, Day CM, Erritzoe D, Kaelen $\mathrm{M}$, et al. Psilocybin with psychological support for treatment-resistant depression: an open-label feasibility study. Lancet Psychiatry. (2016) 3:61927. doi: 10.1016/S2215-0366(16)30065-7

7. Palhano-Fontes F, Barreto D, Onias H, Andrade KC, Novaes MM, Pessoa JA, et al. Rapid antidepressant effects of the psychedelic ayahuasca in treatmentresistant depression: a randomized placebo-controlled trial. Psychol Med. (2019) 49:655-63. doi: 10.1017/S0033291718001356

8. Davis AK, Barrett FS, May DG, Cosimano MP, Sepeda ND, Johnson MW, et al. Effects of psilocybin-assisted therapy on major depressive disorder: a randomized clinical trial. JAMA Psychiatry. (2020) 78: 481-9. doi: 10.1001/jamapsychiatry.2020.3285

9. Johnson MW, Garcia-Romeu A, Cosimano MP, Griffiths RR. Pilot study of the 5-HT2AR agonist psilocybin in the treatment of tobacco addiction. $J$ Psychopharmacol. (2014) 28:983-92. doi: 10.1177/0269881114548296

10. Grob CS, Danforth AL, Chopra GS, Hagerty M, McKay CR, Halberstadt $\mathrm{AL}$, et al. Pilot study of psilocybin treatment for anxiety in patients

\section{ETHICS STATEMENT}

The studies involving human participants were reviewed and approved by this study was conducted in accordance with the Helsinki declaration and approved by the Committee for Research Ethics at the Jose Maria Ramos Mejia General Hospital (Buenos Aires, Argentina), accredited by the Central Committee of Ethics, Ministry of Health of the Autonomous City of Buenos Aires, Argentina. The patients/participants provided their written informed consent to participate in this study.

\section{AUTHOR CONTRIBUTIONS}

ET, FZ, and CP designed the experiment, conducted the experiments, analyzed the data, and wrote the manuscript. FC, YS, and LF conducted the experiments and analyzed the data. CR conducted the experiments and the psychiatric interviews with the participants. All authors contributed to the article and approved the submitted version.

\section{FUNDING}

This work was supported by funding from Agencia Nacional De Promocion Cientifica y Tecnologica (Argentina), grant PICT-2018-03103 and PICT-2019-02294. with advanced-stage cancer. Arch Gen Psychiatry. (2011) 68:71-8. doi: 10.1001/archgenpsychiatry.2010.116

11. Griffiths RR, Johnson MW, Carducci MA, Umbricht A, Richards WA, Richards BD, et al. Psilocybin produces substantial and sustained decreases in depression and anxiety in patients with life-threatening cancer: a randomized double-blind trial. J Psychopharmacol. (2016) 30:118197. doi: $10.1177 / 0269881116675513$

12. Johnson MW, Richards WA, Griffiths RR. Human hallucinogen research: guidelines for safety. J Psychopharmacol. (2008) 22:603-20. doi: 10.1177/0269881108093587

13. Sessa B. The 21st century psychedelic renaissance: heroic steps forward on the back of an elephant. Psychopharmacology. (2018) 235:551-60. doi: 10.1007/s00213-017-4713-7

14. Griffiths RR, Richards WA, McCann U, Jesse R. Psilocybin can occasion mystical-type experiences having substantial and sustained personal meaning and spiritual significance. Psychopharmacology. (2006) 187:268-83. doi: 10.1007/s00213-006-0457-5

15. Griffiths RR, Richards WA, Johnson MW, McCann UD, Jesse R. Mysticaltype experiences occasioned by psilocybin mediate the attribution of personal meaning and spiritual significance 14 months later. J Psychopharmacol. (2008) 22:621-32. doi: 10.1177/0269881108094300

16. Barrett FS, Griffiths RR. Classic hallucinogens and mystical experiences: phenomenology and neural correlates. In: Halberstadt AL, Vollenweider FX, Nichols DE (eds). Behavioral Neurobiology of Psychedelic Drugs. Berlin; Heidelberg: Springer (2017). p. 393-430. doi: 10.1007/7854_2017_474

17. Johnson MW, Hendricks PS, Barrett FS, Griffiths RR. Classic psychedelics: an integrative review of epidemiology, therapeutics, mystical experience, and brain network function. Pharmacol Therapeut. (2019) 197:83-102. doi: 10.1016/j.pharmthera.2018.11.010

18. Barrett FS, Johnson MW, Griffiths RR. Validation of the revised Mystical Experience Questionnaire in experimental sessions with psilocybin. $J$ Psychopharmacol. (2015) 29:1182-90. doi: 10.1177/0269881115609019

19. Griffiths RR, Johnson MW, Richards WA, Richards BD, McCann U, Jesse R. Psilocybin occasioned mystical-type experiences: immediate 
and persisting dose-related effects. Psychopharmacology. 218:649-65. doi: 10.1007/s00213-011-2358-5

20. Roseman L, Nutt DJ, Carhart-Harris RL. Quality of acute psychedelic experience predicts therapeutic efficacy of psilocybin for treatment-resistant depression. Front Pharmacol. (2018) 8:974. doi: 10.3389/fphar.2017.00974

21. Garcia-Romeu AR, Griffiths RW, Johnson M. Psilocybin-occasioned mystical experiences in the treatment of tobacco addiction. Curr Drug Abuse Rev. (2014) 7:157-64. doi: 10.2174/1874473708666150107121331

22. Liechti ME, Dolder PC, Schmid Y. Alterations of consciousness and mysticaltype experiences after acute LSD in humans. Psychopharmacology. (2017) 234:1499-510. doi: 10.1007/s00213-016-4453-0

23. Griffiths RR, Hurwitz ES, Davis AK, Johnson MW, Jesse R. Survey of subjective "God encounter experiences": comparisons among naturally occurring experiences and those occasioned by the classic psychedelics psilocybin, LSD. ayahuasca, or DMT. PLoS ONE. (2019) 14:e0214377. doi: 10.1371/journal.pone.0214377

24. Carhart-Harris RL, Roseman L, Haijen E, Erritzoe D, Watts R, Branchi I, et al. Psychedelics and the essential importance of context. J Psychopharmacol. (2018) 32:725-31. doi: 10.1177/0269881118754710

25. Haijen EC, Kaelen M, Roseman L, Timmermann C, Kettner H, Russ S, et al. Predicting responses to psychedelics: a prospective study. Front Pharmacol. (2018) 9:897. doi: 10.3389/fphar.2018.00897

26. Aday JS, Davis AK, Mitzkovitz CM, Bloesch EK, Davoli CC. Predicting reactions to psychedelic drugs: a systematic review of states and traits related to acute drug effects. ACS Pharmacol Transl Sci. (2021). 4:424-35. doi: 10.1021/acsptsci.1c00014

27. Studerus E, Gamma A, Kometer M, Vollenweider FX. Prediction of psilocybin response in healthy volunteers. PLoS ONE. (2012) 7:e30800. doi: 10.1371/journal.pone.0030800

28. Cakic V, Potkonyak J, Marshall A. Dimethyltryptamine (DMT): subjective effects and patterns of use among Australian recreational users. Drug Alcohol Depend. (2010) 111:30-7. doi: 10.1016/j.drugalcdep.2010.03.015

29. Winstock AR, Kaar S, Borschmann R. Dimethyltryptamine (DMT): prevalence, user characteristics and abuse liability in a large global sample. J Psychopharmacol. (2014) 28:49-54. doi: 10.1177/0269881113513852

30. Szara ST. Dimethyltryptamin: Its metabolism in man, the relation of its psychotic effect to the serotonin metabolism. Experientia. (1956) 12:4412. doi: 10.1007/BF02157378

31. Strassman RJ, Qualls CR, Uhlenhuth EH, Kellner R. Dose-response study of N. N-dimethyltryptamine in humans: II. Subjective effects and preliminary results of a new rating scale. Arch Gen Psychiatry. (1994) 51:98108. doi: 10.1001/archpsyc.1994.03950020022002

32. Shulgin A, Shulgin A. TIHKAL: The Continuation. Transform Press, Berkeley, CA. (1997).

33. Shamay-Tsoory SG, Mendelsohn A. Real-life neuroscience: an ecological approach to brain and behavior research. Perspect Psychol Sci. (2019) 14:84159. doi: 10.1177/1745691619856350

34. Spielberger CD. State-trait anxiety inventory. In: Weiner IB, Craighead WE (eds). The Corsini Encyclopedia of Psychology. Hoboken, NJ: John Wiley \& Sons, Inc. (2010). doi: 10.1002/9780470479216.corpsy0943

35. Ott J. Pharmahuasca: human pharmacology of oral DMT plus harmine. J Psychoactive Drugs. (1999) 31:171-7. doi: 10.1080/02791072.1999.1047 1741

36. Studerus E, Gamma A, Vollenweider FX. Psychometric evaluation of the altered states of consciousness rating scale (OAV). PLoS ONE. (2010) 5:e12412. doi: 10.1371/journal.pone.0012412

37. Greyson B. The near-death experience scale. J Nervous Mental Dis. (1983) 171:369-75. doi: 10.1097/00005053-198306000-00007

38. John OP, Srivastava S. The Big Five trait taxonomy: history, measurement, theoretical perspectives. Handb Pers Theory Res. (1999) 2:102-38.

39. Tellegen A, Atkinson G. Openness to absorbing and self-altering experiences ("absorption"), a trait related to hypnotic susceptibility. J Abnormal Psychol. (1974) 83:268. doi: 10.1037/h0036681

40. Delorme A, Makeig S. EEGLAB: an open source toolbox for analysis of singletrial EEG dynamics including independent component analysis. J Neurosci Methods. (2004) 134:9-21. doi: 10.1016/j.jneumeth.2003.10.009
41. Tadel F, Baillet S, Mosher JC, Pantazis D, Leahy RM. Brainstorm: a userfriendly application for MEG/EEG analysis. Comput Intell Neurosci. (2011) 2011:879716. doi: 10.1155/2011/879716

42. Dale AM, Liu AK, Fischl BR, Buckner RL, Belliveau JW, Lewine JD, et al. Dynamic statistical parametric mapping: combining fMRI and MEG for high-resolution imaging of cortical activity. Neuron. (2000) 26:5567. doi: 10.1016/S0896-6273(00)81138-1

43. Tzourio-Mazoyer N, Landeau B, Papathanassiou D, Crivello F, Etard O, Delcroix N, et al. Automated anatomical labeling of activations in SPM using a macroscopic anatomical parcellation of the MNI MRI single-subject brain. Neuroimage. (2002) 15:273-89. doi: 10.1006/nimg.2001.0978

44. Benjamini Y, Hochberg Y. Controlling the false discovery rate: a practical and powerful approach to multiple testing. J R Stat Soc Ser B. (1995) 57:289300. doi: 10.1111/j.2517-6161.1995.tb02031.x

45. Liaw A, Wiener M. Classification and regression by randomForest. $R$ News. (2002) 2:18-22.

46. Pedregosa F, Varoquaux G, Gramfort A, Michel V, Thirion B, Grisel O, et al. Scikit-learn: machine learning in Python. J Mach Learn Res. (2011) 12:282530 .

47. Timmermann C, Roseman L, Schartner M, Milliere R, Williams LT, Erritzoe D, et al. Neural correlates of the DMT experience assessed with multivariate EEG. Sci Rep. (2019) 9:1-13. doi: 10.1038/s41598-019-51974-4

48. Schenberg EE, Alexandre JFM, Filev R, Cravo AM, Sato JR, Muthukumaraswamy SD, et al. Acute biphasic effects of ayahuasca. PLoS ONE. (2015) 10:e0137202. doi: 10.1371/journal.pone.0137202

49. Klimesch W. EEG alpha and theta oscillations reflect cognitive and memory performance: a review and analysis. Brain Res Rev. (1999) 29:16995. doi: 10.1016/S0165-0173(98)00056-3

50. Sauseng P, Klimesch W, Schabus M, Doppelmayr M. Frontoparietal EEG coherence in theta and upper alpha reflect central executive functions of working memory. Int J Psychophysiol. (2005) 57:97-103. doi: 10.1016/j.ijpsycho.2005.03.018

51. Demiralp T, Bayraktaroglu Z, Lenz D, Junge $S$, Busch NA, Maess B, et al. Gamma amplitudes are coupled to theta phase in human EEG during visual perception. Int J Psychophysiol. (2007) 64:24-30. doi: 10.1016/j.ijpsycho.2006.07.005

52. Sauseng P, Griesmayr B, Freunberger R, Klimesch W. Control mechanisms in working memory: a possible function of EEG theta oscillations. Neurosci Biobehav Rev. (2010) 34:1015-22. doi: 10.1016/j.neubiorev.2009.12.006

53. Smigielski L, Kometer M, Scheidegger M, Krähenmann R, Huber T, Vollenweider FX. Characterization and prediction of acute and sustained response to psychedelic psilocybin in a mindfulness group retreat. Sci Rep. (2019) 9:1-13. doi: 10.1038/s41598-019-50612-3

54. van Son D, de Rover M, De Blasio FM, van der Does W, Barry RJ, Putman P. Electroencephalography theta/beta ratio covaries with mind wandering and functional connectivity in the executive control network. Ann N Y Acad Sci. (2019) 1452:52. doi: 10.1111/nyas.14180

55. van Son D, De Blasio FM, Fogarty JS, Angelidis A, Barry RJ, Putman P. Frontal EEG theta/beta ratio during mind wandering episodes. Biol Psychol. (2019) 140:19-27. doi: 10.1016/j.biopsycho.2018.11.003

56. Braboszcz C, Delorme A. Lost in thoughts: neural markers of low alertness during mind wandering. Neuroimage. (2011) 54:3040-7. doi: 10.1016/j.neuroimage.2010.10.008

57. Qin J, Perdoni C, He B. Dissociation of subjectively reported and behaviorally indexed mind wandering by EEG rhythmic activity. PLoS ONE. (2011) 6:e23124. doi: 10.1371/journal.pone.0023124

58. Soffer-Dudek N, Todder D, Shelef L, Deutsch I, Gordon S. A neural correlate for common trait dissociation: decreased EEG connectivity is related to dissociative absorption. J Pers. (2019) 87:295-309. doi: 10.1111/jopy.12391

59. Groot JM, Boayue NM, Csifcsák G, Boekel W, Huster R, Forstmann $\mathrm{BU}$, et al. Probing the neural signature of mind wandering with simultaneous fMRI-EEG and pupillometry. Neuroimage. (2021) 224:117412. doi: 10.1016/j.neuroimage.2020.117412

60. Andersen SB, Moore RA, Venables L, Corr PJ. Electrophysiological correlates of anxious rumination. Int J Psychophysiol. (2009) 71:15669. doi: 10.1016/j.ijpsycho.2008.09.004 
61. Steinvorth S, Wang C, Ulbert I, Schomer D, Halgren E. Human entorhinal gamma and theta oscillations selective for remote autobiographical memory. Hippocampus. (2010) 20:166-73. doi: 10.1002/hipo.20597

62. Lavallee CF, Persinger MA. A LORETA study of mental time travel: similar and distinct electrophysiological correlates of re-experiencing past events and pre-experiencing future events. Conscious Cogn. (2010) 19:103744. doi: 10.1016/j.concog.2010.06.008

63. So WK, Wong SW, Mak JN, Chan RH. An evaluation of mental workload with frontal EEG. PLoS ONE. (2017) 12:e0174949. doi: 10.1371/journal.pone.0174949

64. Lagopoulos J, Xu J, Rasmussen I, Vik A, Malhi GS, Eliassen CF, et al. Increased theta and alpha EEG activity during nondirective meditation. J Alternat Complement Med. (2009) 15:1187-92. doi: 10.1089/acm.2009.0113

65. Xue SW, Tang YY, Tang R, Posner MI. Short-term meditation induces changes in brain resting EEG theta networks. Brain Cogn. (2014) 87:16. doi: 10.1016/j.bandc.2014.02.008

66. Lomas T, Ivtzan I, Fu CH. A systematic review of the neurophysiology of mindfulness on EEG oscillations. Neurosci Biobehav Rev. (2015) 57:40110. doi: 10.1016/j.neubiorev.2015.09.018

67. Harne BP, Hiwale AS. EEG spectral analysis on OM mantra meditation: a pilot study. Appl Psychophysiol Biofeedback. (2018) 43:123-9. doi: 10.1007/s10484-018-9391-7

68. Scheeringa R, Bastiaansen MC, Petersson KM, Oostenveld R, Norris DG, Hagoort P. Frontal theta EEG activity correlates negatively with the default mode network in resting state. Int J Psychophysiol. (2008) 67:24251. doi: 10.1016/j.ijpsycho.2007.05.017

69. Zuure MB, Hinkley LB, Tiesinga PH, Nagarajan SS, Cohen MX. Multiple midfrontal thetas revealed by source separation of simultaneous MEG and EEG. J Neurosci. (2020) 40:7702-13. doi: 10.1523/JNEUROSCI.0321-20.2020
70. Berkovich-Ohana A, Glicksohn J. Meditation, absorption, transcendent experience, and affect: tying it all together via the consciousness state space (CSS) model. Mindfulness. (2017) 8:68-77. doi: 10.1007/s12671-0150481-9

71. Pallavicini, C., Cavanna, F., Zamberlan, F., de la Fuente, L. A., Ilksoy, Y., Perl, Y. S., Arias, M., Romero, C., Carhart-Harris, R., Timmermann, C., Tagliazucchi, E. (2021). Neural and subjective effects of inhaled $\mathrm{N}, \mathrm{N}$-dimethyltryptamine in natural settings. J Psychopharm. 35:406-420.

Conflict of Interest: The authors declare that the research was conducted in the absence of any commercial or financial relationships that could be construed as a potential conflict of interest.

Publisher's Note: All claims expressed in this article are solely those of the authors and do not necessarily represent those of their affiliated organizations, or those of the publisher, the editors and the reviewers. Any product that may be evaluated in this article, or claim that may be made by its manufacturer, is not guaranteed or endorsed by the publisher.

Copyright (c) 2021 Tagliazucchi, Zamberlan, Cavanna, de la Fuente, Romero, Sanz Perl and Pallavicini. This is an open-access article distributed under the terms of the Creative Commons Attribution License (CC BY). The use, distribution or reproduction in other forums is permitted, provided the original author(s) and the copyright owner(s) are credited and that the original publication in this journal is cited, in accordance with accepted academic practice. No use, distribution or reproduction is permitted which does not comply with these terms. 